\title{
Regulation of hypoxia-induced angiogenesis: a chaperone escorts VEGF to the dance
}

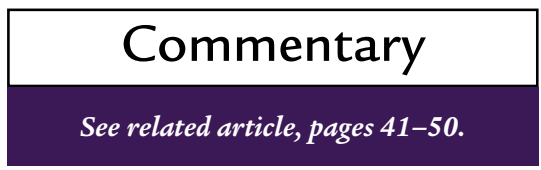

\author{
Gregg L. Semenza \\ Institute of Genetic Medicine, The Johns Hopkins University School of Medicine, CMSC-1004, 600 N. Wolfe Street, Baltimore, \\ Maryland 21287-3914, USA. Fax: (410) 955-0484; E-mail: gsemenza@jhmi.edu. \\ J. Clin. Invest. 108:39-40 (2001). DOI:10.1172/JCI200113374.
}

In contrast to invertebrate species, in which oxygen can be adequately transported to all cells by diffusion, vertebrates, with their larger body size, require a circulatory system that is specialized for oxygen delivery. When systemic oxygen delivery is inadequate, cardiac output increases and red blood cell production is augmented. However, when oxygen delivery is impaired locally, as in a tissue in which perfusion has been interrupted by injury or vascular stenosis, a primary response is angiogenesis, the sprouting of new blood vessels.

The complex process of angiogenesis (reviewed in refs. 1-3) begins when cells within a tissue respond to hypoxia by increasing their production of VEGF. VEGF is secreted and binds to cognate receptor tyrosine kinases (VEGFR1 and VEGFR2) located on the surface of vascular endothelial cells. Receptor ligation triggers a cascade of intracellular signaling pathways that initiate angiogenesis. The clinical importance of this biological process has become increasingly apparent over the last decade, and angiogenesis now represents a major focus for novel therapeutic approaches to the prevention and treatment of multiple diseases, most notably ischemic cardiovascular disease and cancer (1-3).

If angiogenesis is the big dance, then VEGF is the belle of the ball, and hundreds of investigators are now studying the regulation and biological consequences of its expression (reviewed in ref. 4). Although VEGF expression represents only one component of the complex angiogenic response and VEGF administration alone may not be sufficient to effect therapeutic angiogenesis, VEGF can nevertheless be considered a critical activator of vascular endothelial cells. Establishing the molecular mechanisms regulating VEGF activity is therefore essential for understanding angiogenesis and how it may be targeted clinically. When cells are subjected to hypoxia, VEGF mRNA expression is induced as a result of both increased mRNA transcription and decreased mRNA degradation (5-7). Whereas the rate of translation initiation is globally decreased in hypoxic cells, VEGF mRNAs contain an internal ribosome entry site (IRES) that ensures efficient VEGF protein synthesis under hypoxic conditions (8). In addition, expression of VEGFR1 (FLT-1) mRNA is hypoxiainducible (9), indicating that the endothelium must sense hypoxia in order to be maximally receptive to VEGF stimulation. Endothelial cell expression of VEGFR2 (KDR) protein is also induced in ischemic tissue via a paracrine mechanism (10). Finally, placental growth factor (PlGF), which binds to VEGFR1 (4), is induced by hypoxia in many tissues and in combination with VEGF has synergistic effects on angiogenesis (11).

Despite the impressive complexity of the molecular responses to hypoxia that are known to be involved in VEGF signaling, our understanding of this process continues to grow. In this issue of the JCI, Ozawa et al. (12) report studies indicating that the chaperone protein ORP150 (oxygenregulated protein of $150 \mathrm{kDa}$ ) is required for the intracellular transport of VEGF protein from the endoplasmic reticulum to the Golgi apparatus for subsequent secretion. ORP150 was first identified and purified as a protein expressed in primary cultures of astrocytes exposed to hypoxia (13), and, like VEGF, ORP150 mRNA expression is also hypoxia-inducible (12). To confirm the physiological and clinical relevance of their findings, Ozawa et al. demonstrate coexpression of ORP150 and VEGF in human wound macrophages. Administration of adenovirus encoding ORP150 to wounds of diabetic mice accelerated neovascularization and repair in vivo. ORP150 overexpression increased VEGF protein secretion in hypoxic tissue culture cells, whereas expression of ORP150 antisense RNA resulted in accumulation of VEGF within the endoplasmic reticulum. The data indicate that ORP150 is required for VEGF secretion and that in hypoxic cells increased levels of VEGF necessitate a corresponding increase in ORP150 expression. If this is true, then one would expect ORP150 to be coexpressed with VEGF in ischemic and neoplastic cells. Stimulation or inhibition of ORP150 expression or activity would then have a corresponding effect on VEGF and angiogenesis, hypotheses that will undoubtedly be tested in the near future.

Taken together, the results of studies conducted over the last six years have delineated complex regulation of VEGF activity by tissue oxygen concentrations (Table 1). How is this response orchestrated at the molecular level? Hypoxia-inducible factor 1 (HIF1 ) is responsible for the transcriptional activation of the $V E G F$ gene in hypoxic cells by binding to a hypoxia response element located $1 \mathrm{~kb} 5^{\prime}$ to the transcription initiation site $(14,15)$. Once transcribed, VEGF mRNA is bound by HuR and other proteins that inhibit its degradation under hypoxic conditions (16). It is not known

\section{Table 1}

Regulation of VEGF signaling

in hypoxic/ischemic tissue

\begin{tabular}{lc} 
Response & Regulator \\
VEGF gene transcription & HIF-1 \\
VEGF mRNA stability & HuR \\
VEGF mRNA translation via IRES & $?$ \\
VEGF protein transport & ORP150 \\
VEGFR1 gene transcription & HIF-1 \\
VEGFR2 protein expression & $?$ \\
PIGF mRNA expression & $?$ \\
\hline
\end{tabular}


whether a specific translation initiation factor exists that recognizes the IRES and whether expression of this factor is induced by hypoxia. HIF-1 has also been implicated in the induction of FLT-1 mRNA expression in hypoxic endothelial cells (9). Since ORP150 mRNA expression is induced by hypoxia, the question arises whether HIF-1 activates ORP150 transcription. Indeed, HIF-1 may coordinately regulate VEGF signaling at multiple levels (Table 1), similar to its coordinate regulation of more than a dozen genes encoding the glucose transporters and glycolytic enzymes that provide metabolic adaptation to hypoxia (15). The recent finding that brain-derived neurotrophic factor is an ORP150-regulated protein in hypoxic neurons (17) also suggests that ORP150 may function as a chaperone for other HIF-1-regulated and/or hypoxia-inducible growth factors such as angiopoietin 2, erythropoietin, and IGF-2. In addition to delineating elegant programs of molecular regulation, testing these hypotheses may provide information as to which molecules are best suited to induce therapeutic angiogenesis, with the assumption being that regulatory control factors may have the most pleiotropic effects and thus best recapitulate the complex physiologic responses evoked by hypoxia.

Ozawa et al. (12) chose wound healing as the clinical condition in which to demonstrate the relevance of ORP150 as a mediator of VEGF secretion in vivo. This is an interesting choice because experimental and clinical data suggest that the early wound is not hypoxic (18) and that hyperoxia stimulates wound angiogenesis and healing
(19). VEGF transcription by wound macrophages may be stimulated by reactive oxygen species that are generated by activated neutrophils (20). The transcription factor mediating this response has not been identified. However, HIF-1 activity is induced by inflammatory cytokines such as IL-1 and TNF- $\alpha$ (21) which are generated at high levels in early wounds (18), thus providing a mechanism for VEGF production mediated by HIF-1 within nonhypoxic wounds (22). These observations point to the potential importance of shared transcriptional mechanisms, involving HIF-1 and/or other factors, that would couple VEGF and ORP150 expression regardless of the nature of the inciting stimulus. Thus, while the more than 4700 publications listed in the NCBI PubMed database suggest that VEGF has come of age, Ozawa et al. have shown that it still requires a chaperone.

1. Blau, H.M., and Banfi, A. 2001. The well-tempered vessel. Nat. Med. 7:532-534.

2. Carmeliet, P., and Jain, R.K. 2000. Angiogenesis in cancer and other diseases. Nature. 407:249-257.

3. Yancopoulos, G.D., et al. 2000. Vascular-specific growth factors and blood vessel formation. Nature. 407:242-248.

4. Ferrara, N. 2001. Role of vascular endothelial growth factor in regulation of physiological angiogenesis. Am. J. Physiol. 280:C1358-C1366

5. Levy, A.P., Levy, N.S., Wegner, S., and Goldberg, M.A. 1995. Transcriptional regulation of the rat vascular endothelial growth factor gene by hypoxia. J. Biol. Chem. 270:13333-13340.

6. Ikeda, E., Achen, M.G., Breier, G., and Risau, W. 1995. Hypoxia-induced transcriptional activation and increased mRNA stability of vascular endothelial growth factor in C6 glioma cells. J. Biol. Chem. 270:19761-19766.

7. Levy, A.P., Levy, N.S., and Goldberg, M.A. 1996. Post-transcriptional regulation of vascular endothelial growth factor by hypoxia. J. Biol. Chem. 271:2746-2753.

8. Stein, I., et al. 1998. Translation of vascular endothelial growth factor mRNA by internal ribosome entry: implications for translation under hypoxia. Mol. Cell. Biol. 18:3112-3119.

9. Gerber, H.P., Condorelli, F., Park, J., and Ferrara, N. 1997. Differential transcriptional regulation of the two vascular endothelial growth factor receptor genes. Flt-1, but not Flk-1/KDR, is upregulated by hypoxia. J. Biol. Chem. 272:23659-23667.

10. Carmeliet, P., et al. 2001. Synergism between vascular endothelial growth factor and placental growth factor contributes to angiogenesis and plasma extravasation in pathological conditions. Nat. Med. 7:575-583

11. Brogi, E., et al. 1996. Hypoxia-induced paracrine regulation of vascular endothelial growth factor receptor expression. J. Clin. Invest. 97:469-476.

12. Ozawa, K., et al. 2001. Expression of the oxygenregulated protein ORP150 accelerates wound healing by modulating intracellular VEGF transport. J. Clin. Invest. 108:41-50.

13. Kuwabara, K., et al. 1996. Purification and characterization of a novel stress protein, the $150-\mathrm{kDa}$ oxygen-regulated protein (ORP150), from cultured rat astrocytes and its expression in ischemic mouse brain. J. Biol. Chem. 271:5025-5032.

14. Forsythe, J.A., et al. 1996. Activation of vascular endothelial growth factor gene transcription by hypoxia-inducible factor $1 . \mathrm{Mol}$. Cell. Biol. 16:4604-4613.

15. Iyer, N.V., et al. 1998. Cellular and developmen tal control of $\mathrm{O}_{2}$ homeostasis by hypoxiainducible factor $1 \alpha$. Genes Dev. 12:149-162.

16. Levy, N.S., Chung, S., Furneaux, H., and Levy, A.P 1998. Hypoxic stabilization of vascular endothelial growth factor mRNA by the RNA-binding protein HuR. J. Biol. Chem. 273:6417-6423.

17. Tamatani, M., et al. 2001. ORP150 protects against hypoxia/ischemia-induced neuronal death. Nat. Med. 7:317-323.

18. Haroon, Z.A., Raleigh, J.A., Greenberg, C.S., and Dewhirst, M.W. 2000. Early wound healing exhibits cytokine surge without evidence of hypoxia. Ann. Surg. 231:137-147.

19. Sheikh, A.Y., et al. 2000. Effect of hyperoxia on vascular endothelial growth factor levels in a wound model. Arch. Surg. 135:1293-1297.

20. Cho, M., Hunt, T.K., and Hussain, M.Z. 2001 Hydrogen peroxide stimulates macrophage vascular endothelial growth factor release. Am. J. Physiol. 280:H2357-H2363.

21. Hellwig-Burgel, T., Rutkowski, K., Metzen, E. Fandrey, J., and Jelkmann, W. 1999. Interleukin$1 \beta$ and tumor necrosis factor- $\alpha$ stimulate DNA binding of hypoxia-inducible factor-1. Blood. 94:1561-1567.

22. Elson, D.A., Ryan, H.E., Snow, J.W., Johnson, R. and Arbeit, J.M. 2000. Coordinate up-regulation of hypoxia inducible factor (HIF)- $1 \alpha$ and HIF- 1 target genes during multi-stage epidermal carcinogenesis and wound healing. Cancer Res. 60:6189-6195. 